\title{
AN ULTRADEEP SURVEY FOR IRREGULAR SATELLITES OF URANUS: LIMITS TO COMPLETENESS ${ }^{1,2}$
}

\author{
Scott S. Sheppard, ${ }^{3}$ David Jewitt, and Jan Kleyna \\ Institute for Astronomy, University of Hawaii, 2680 Woodlawn Drive, Honolulu, HI 96822; \\ sheppard@dtm.ciw.edu, jewitt@ifa.hawaii.edu,kleyna@ifa.hawaii.edu \\ Received 2004 August 4; accepted 2004 September 21
}

\begin{abstract}
We present a deep optical survey of Uranus's Hill sphere for small satellites. The $8 \mathrm{~m}$ Subaru Telescope was used to survey about 3.5 square degrees with a $50 \%$ detection efficiency at limiting red magnitude $m_{R}=26.1$. This magnitude corresponds to objects that are about $7 \mathrm{~km}$ in radius (assuming an albedo of 0.04 ). We detected (without prior knowledge of their positions) all previously known outer satellites and discovered two new irregular satellites (S/2001 U2 and S/2003 U3). The two inner satellites Titania and Oberon were also detected. One of the newly discovered bodies (S/2003 U3) is the first known irregular prograde satellite of the planet. The population, size distribution, and orbital parameters of Uranus's irregular satellites are remarkably similar to those of the irregular satellites of gas giant Jupiter. Both have shallow size distributions (power-law indices $q \sim 2$ for radii larger than $7 \mathrm{~km}$ ) with no correlation between the sizes of the satellites and their orbital parameters. However, unlike those of Jupiter, Uranus's irregular satellites do not appear to occupy tight, distinct dynamical groups in semimajor-axis versus inclination phase space. Two groupings in semimajor-axis versus eccentricity phase space appear to be statistically significant.
\end{abstract}

Key words: solar system: general — planets and satellites: individual (Uranus)

\section{INTRODUCTION}

Planetary satellites are confined to the space in which the planet's gravitational force dominates over that of the Sun. This region is known as the Hill sphere, the radius of which, $r_{\mathrm{H}}$, is given by

$$
r_{\mathrm{H}}=a_{p}\left(\frac{m_{p}}{3 M_{\odot}}\right)^{1 / 3}
$$

where $a_{p}$ and $m_{p}$ are the semimajor axis and mass of the planet and $M_{\odot}$ is the mass of the Sun. Table 1 lists the Hill sphere radius and projected area for each of the giant planets.

The giant planets possess two distinct types of satellite (Peale 1999). Regular satellites are found within about $0.05 r_{\mathrm{H}}$ and are tightly bound to their planet. They have nearly circular, prograde orbits with low inclinations. Regular satellites likely formed within a circumplanetary disk of gas and dust around the giant planets as part of the planetary formation process itself. In contrast, irregular satellites are found up to $0.65 r_{\mathrm{H}}$ from their host planets and have moderate-to-high eccentricities and inclinations with prograde or retrograde orbits. Irregular satellites cannot have formed in their present orbits and are likely products of early capture from heliocentric orbit (Kuiper 1956; Pollack et al. 1979). Table 1 lists the currently known populations of irregular satellites for each giant planet as of 2004 August 1.

Burns (1986) offered a definition of irregular satellites as those satellites that are far enough from their parent planet that

\footnotetext{
${ }^{1}$ Based largely on data collected at the Subaru Telescope, which is operated by the National Astronomical Observatory of Japan.

${ }^{2}$ Based in part on observations obtained at the Gemini Observatory, which is operated by the Association of Universities for Research in Astronomy, Inc., under a cooperative agreement with the NSF on behalf of the Gemini partnership.

${ }^{3}$ Current address: Department of Terrestrial Magnetism, Carnegie Institution of Washington, 5241 Broad Branch Road, NW, Washington, DC 20015.
}

the precession of their orbital plane is primarily controlled by the Sun instead of the planet's oblateness. In other words, the satellite's inclination is fixed relative to the planet's orbital plane instead of the planet's equator. By this definition, any satellite with a semimajor axis larger than the critical value $a_{\text {crit }} \sim$ $\left[2 J_{2} r_{p}^{2} a_{p}^{3} m_{p} /\left(1 M_{\odot}\right)\right]^{1 / 5}$ is an irregular satellite (Burns 1986; see Table 1). Here $J_{2}$ is the planet's second gravitational harmonic coefficient and $r_{p}$ is the planet's equatorial radius.

Because of the reversibility of Newton's equations of motion, some sort of energy dissipation is required for permanent satellite capture. The giant planets currently have no efficient mechanism of energy dissipation for satellite capture. During the planet formation epoch, several mechanisms may have operated to capture satellites: (1) gas drag in an extended, primordial planetary atmosphere (Pollack et al. 1979), (2) pulldown capture caused by the mass growth of the planet and consequent expansion of the Hill sphere (Heppenheimer \& Porco 1977), and (3) orbital energy dissipation from collisions or collisionless interactions between asteroids or satellites passing near the planet (Colombo \& Franklin 1971; Tsui 2000). Study of the irregular satellites is important for the insight these objects might provide into the planet formation process.

Core accretion models of planet formation struggle to form Uranus and Neptune within the age of the solar system (Lissauer et al. 1995; Pollack et al. 1996; Boss 2001). Disk instability models do not readily provide the hydrogen- and helium-depleted compositions of the two ice giants (Boss 2001). The massive hydrogen and helium gas giants Jupiter and Saturn likely formed quickly in the protoplanetary disk $\left(\leq 10^{6} \mathrm{yr}\right)$. The less massive, deficient in hydrogen and helium, and more distant ice giants Uranus and Neptune appear to have taken much longer or an altogether different route of formation (Thommes et al. 2002; Boss 2003).

Uranus is noteworthy in the sense that its obliquity exceeds $90^{\circ}$. This compares with the modest obliquities of Jupiter, Saturn and Neptune at $3^{\circ}, 27^{\circ}$, and $30^{\circ}$, respectively. A possible 
TABLE 1

Irregular Satellites of the Planets

\begin{tabular}{|c|c|c|c|c|c|c|}
\hline Planet & Irr. $^{a}$ & Groups $^{\mathrm{b}}$ & $\begin{array}{c}r_{\min } \operatorname{Limit}^{\mathrm{c}} \\
(\mathrm{km})\end{array}$ & $a_{\text {crit }}^{\mathrm{d}}$ & $\begin{array}{l}\text { Hill Radius } \\
\text { (deg) }\end{array}$ & $\begin{array}{l}\text { Hill Area }{ }^{\mathrm{f}} \\
\text { (deg) }\end{array}$ \\
\hline 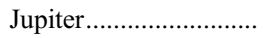 & $55(48 / 7)$ & $\sim 6$ & 1.5 & 0.044 & 4.7 & 70 \\
\hline 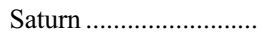 & $14(7 / 7)$ & $\sim 4-5$ & 4 & 0.038 & 3.0 & 28 \\
\hline 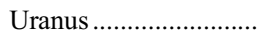 & $9(8 / 1)$ & $\sim 2-3$ & 7 & 0.019 & 1.4 & 6 \\
\hline 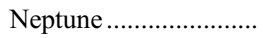 & $7(4 / 3)$ & $\sim 3-4$ & 16 & 0.025 & 1.5 & 7 \\
\hline
\end{tabular}

a Total number of known irregular satellites as of 2004 September 1. Shown in parentheses are the number of known retrograde and prograde irregular satellites (retrograde/prograde).

b Apparent number of dynamical groupings.

c Limiting radii of satellite searches to date.

d Critical semimajor axis in Hill radii. Satellites with semimajor axes larger than this are defined as irregular because their orbits are significantly perturbed by the Sun (Burns 1986).

e The apparent angular Hill sphere radius of the planet at opposition.

$\mathrm{f}$ The Hill sphere area of the planet when at opposition.

cause is that a protoplanet of about 1 Earth mass may have collided with Uranus near the end of its growth phase (Korycansky et al. 1990; Slattery et al. 1992). Greenberg (1974) argued that the current regular satellites must have formed after Uranus's obliquity reached $98^{\circ}$ because their low-inclination prograde orbits would not have adjusted to their current configurations with Uranus. Recently, Brunini et al. (2002) suggested that if Uranus's tilt was created by a giant impact, any satellites beyond about $2 \times 10^{6} \mathrm{~km}$ (i.e., all known irregular satellites of Uranus) would have likely been lost owing to the orbital impulse imparted to Uranus by the impactor. In addition, Beaugé et al. (2002) show that any significant migration by Uranus through a residual planetary disk would have caused its outer satellites to become unstable.

By virtue of its proximity, Jupiter has the best-studied irregular-satellite system (Fig. 1), with 55 irregular satellites

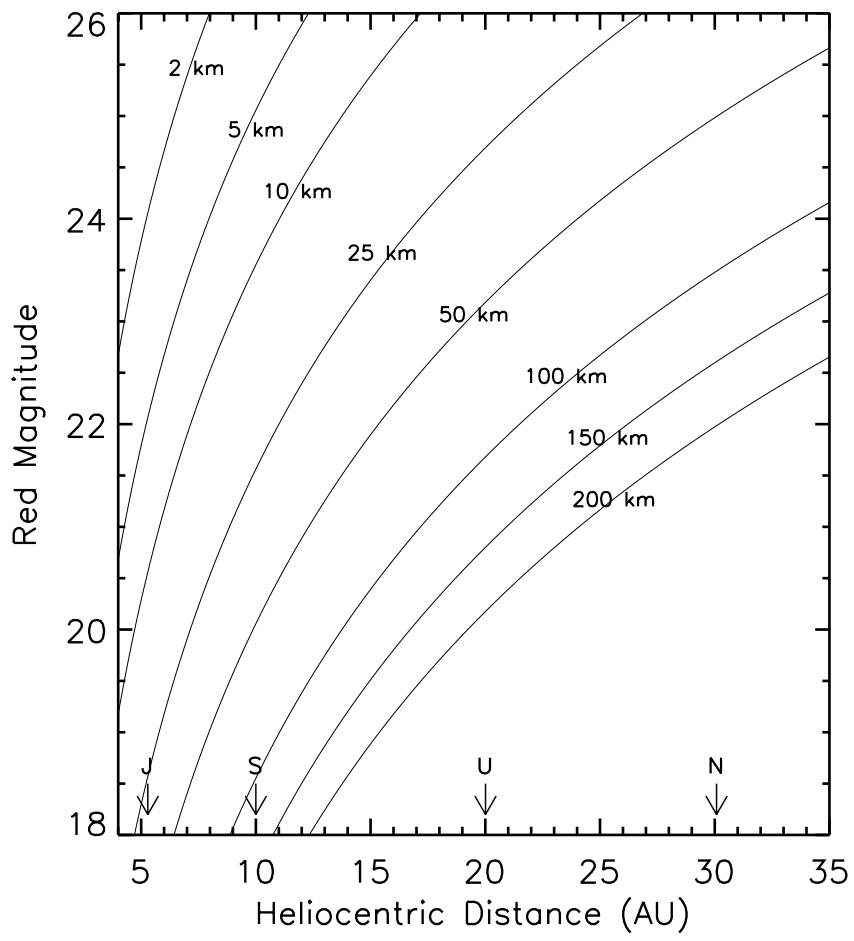

FIG. 1.-Distances of the planets vs. the observable small-body population diameter for a given red magnitude assuming a low (0.04) geometric albedo. The mean semimajor axes of the giant planets Jupiter (J), Saturn (S), Uranus $(\mathrm{U})$, and Neptune $(\mathrm{N})$ are marked. currently known (Sheppard \& Jewitt 2003). In this paper, we ask the question "does the ice giant Uranus have a population of irregular satellites similar to that of gas giant Jupiter?" The greater distance of Uranus requires the use of very deep surveys in order to meaningfully probe the smaller Uranian satellites. Previous surveys near Uranus were conducted using $4 \mathrm{~m}$ class telescopes, including a search of $\sim 5 \mathrm{deg}^{2}$ to limiting red magnitude $m_{R} \sim 24.3$ by Gladman et al. (2000) and one of $\sim 1 \mathrm{deg}^{2}$ to limiting magnitudes in the $m_{R} \sim 25.0-25.4$ range by Kavelaars et al. (2004). In the present work, we used the $8 \mathrm{~m}$ Subaru Telescope and its prime-focus survey camera to survey most of the Hill sphere to a limiting red magnitude $m_{R}=26.1$. Our primary goal was to cover the dynamically stable inner Hill sphere of the planet (radial extent $\sim 0.7 r_{\mathrm{H}}$; see Hamilton \& Krivov 1997) in an unbiased, deep, and uniform survey.

\section{OBSERVATIONS}

We observed the space around Uranus for faint satellites near new Moon on UT 2003 August 29 and 30 with the Subaru $8.2 \mathrm{~m}$ diameter telescope atop Mauna Kea. The geometry of Uranus during the survey is indicated in Table 2. The Suprime-Cam imager has 10 MIT Lincoln Laboratory $2048 \times 4096$ CCDs arranged in a $5 \times 2$ pattern (Miyazaki et al. 2002). Its $15 \mu$ m pixels give a scale of 0.20 pixel $^{-1}$ at prime focus and a field of view that is about $34^{\prime} \times 27^{\prime}$ with the north-south direction aligned with the long axis. Gaps between the chips are about $16^{\prime \prime}$ in the north-south direction and only $3^{\prime \prime}$ in the east-west direction.

Images were obtained with a Kron-Cousins $R$-band filter (central wavelength near $650 \mathrm{~nm}$ ). The images were biassubtracted and then flat-fielded with dome flats taken at the end of each night. During exposures the telescope was autoguided sidereally on field stars. Seeing during the two nights varied between 0". 4 and 0."6 FWHM. Integration times were between 400 and 420 s. Both nights were photometric, and Landolt (1992) standards were used for calibration.

TABLE 2

Geometric Circumstances

\begin{tabular}{cccccc}
\hline \hline $\begin{array}{c}\text { UT } \\
\text { Date }\end{array}$ & $\begin{array}{c}R \\
(\mathrm{AU})\end{array}$ & $\begin{array}{c}\Delta \\
(\mathrm{AU})\end{array}$ & $\begin{array}{c}\alpha \\
(\mathrm{deg})\end{array}$ & $\begin{array}{c}\text { R.A. }^{\mathrm{a}} \\
\left(\operatorname{arcsec} \mathrm{hr}^{-1}\right)\end{array}$ & $\begin{array}{c}\text { Decl. }^{\mathrm{b}} \\
\left(\operatorname{arcsec~hr}^{-1}\right)\end{array}$ \\
\hline 2003 Aug 29........ & 20.031 & 19.024 & 0.25 & -5.7 & -2.1 \\
2003 Aug 30....... & 20.031 & 19.026 & 0.30 & -5.7 & -2.1 \\
\hline
\end{tabular}

a Apparent right ascension motion of Uranus.

b Apparent declination motion of Uranus. 


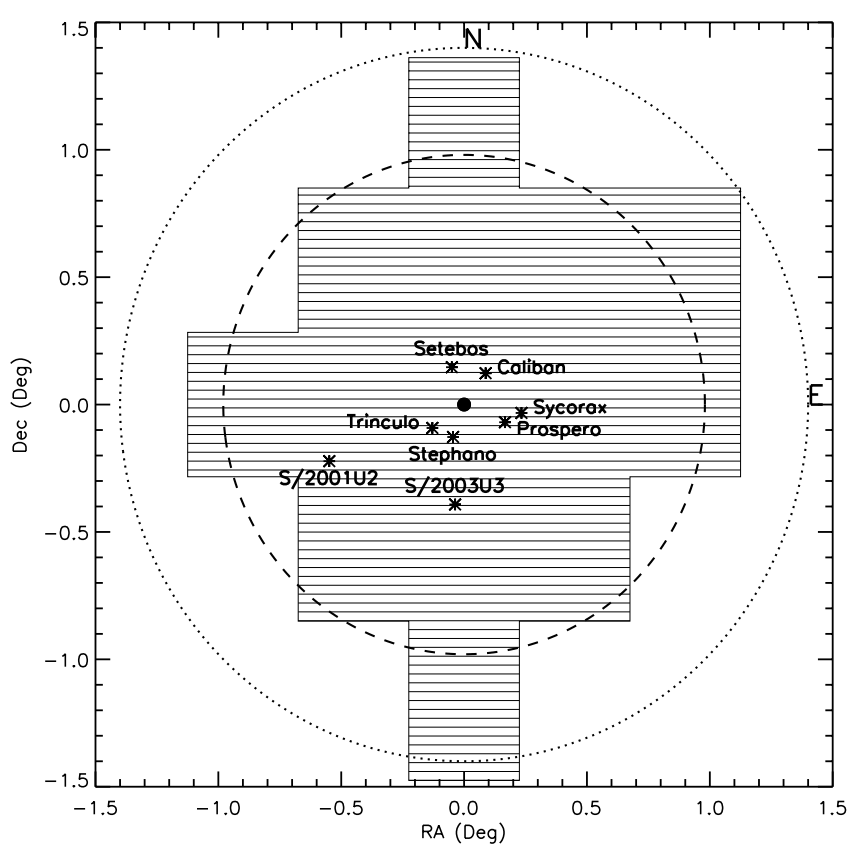

FIG. 2.-Area searched around Uranus for satellites using the $8.2 \mathrm{~m}$ Subaru Telescope. Fourteen fields were imaged on five occasions over two nights (UT 2003 August 29 and 30) for a total of 70 images covering about $3.5 \mathrm{deg}^{2}$. The black dot at the center represents Uranus's position. Uranus was placed in a gap in the mosaic of CCD chips to prevent saturation. The dotted circle shows the Hill sphere of Uranus, while the dashed circle shows the theoretical outer limits of long-term stability for any Uranian satellites $\left(r_{\mathrm{H}} \sim 0.7\right)$. The outer satellites of Uranus are marked at the position of their detection during the survey. In addition, we detected the inner satellites Titania and Oberon (not marked).

The area searched around Uranus for satellites is shown in Figure 2. Fourteen fields were imaged three times each on one night and two times each on the second night for a total of five images per field, or 70 images for the survey. On a given night, images of the same field were separated in time by about 31 minutes. The fields on the second night were centered at the same angular distance from Uranus as on the first night, but the background star fields were different because of Uranus's nonsidereal motion. Approximately $3.5 \mathrm{deg}^{2}$ around Uranus were observed, not accounting for chip gaps and bright stars. We covered $\sim 90 \%$ of the theoretically stable Hill sphere $\left(\sim 0.7 r_{\mathrm{H}}\right)$ for retrograde satellites and near $100 \%$ for the stable Hill sphere $\left(\sim 0.5 r_{\mathrm{H}}\right)$ for prograde satellites (Hamilton \& Krivov 1997).

Satellite confirmation astrometry in the months after discovery was obtained with the Gemini Multi-Object Spectrograph (GMOS) in imaging mode on the $8.1 \mathrm{~m}$ Gemini North telescope atop Mauna Kea (Hook et al. 2003). GMOS has a 5.5 field of view and pixel scale of 0.0727 pixel $^{-1}$. Recoveries were obtained with an $r^{\prime}$ filter based on the Sloan Digital Sky Survey filter set. Images were bias-subtracted and flat-fielded using dome flats. Observations were obtained by a staff astronomer at Gemini in queue scheduling mode.

\section{ANALYSIS}

The Subaru survey observations were obtained when Uranus was near opposition, where the apparent movement is largely parallactic and thus is inversely related to the distance. Objects at the heliocentric distance of Uranus, $R \sim 20 \mathrm{AU}$, will have an apparent motion of about $\sim 6^{\prime \prime} \mathrm{hr}^{-1}\left(\sim 30\right.$ pixels $\left.\mathrm{hr}^{-1}\right)$. At this rate, satellites would have trailed a distance comparable to the FWHM of the seeing during the $400 \mathrm{~s}$ exposures.

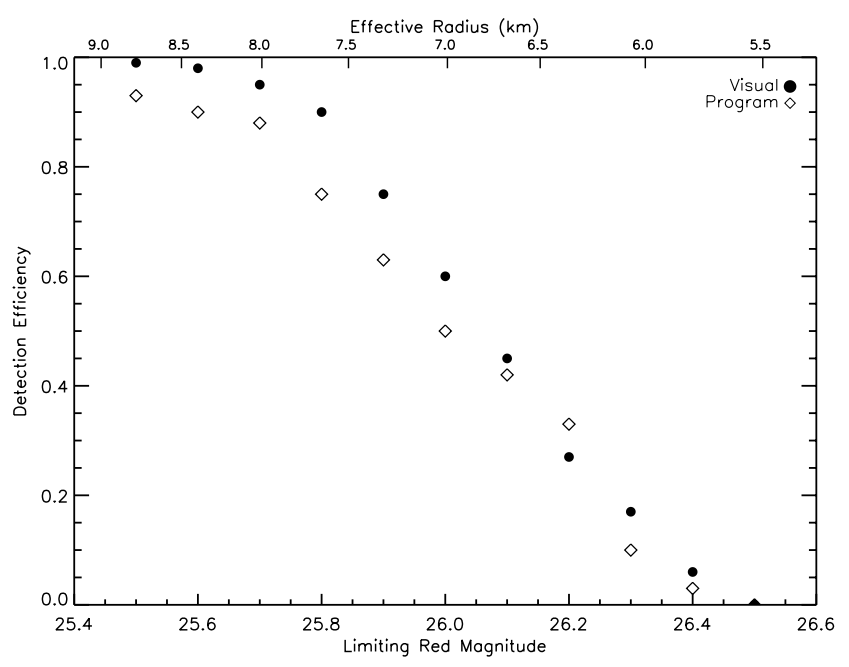

FIG. 3.-Detection efficiency of the Uranus survey vs. the apparent red magnitude. The $50 \%$ detection efficiency is at about $26.1 \mathrm{mag}$ from visual blinking and $26.0 \mathrm{mag}$ from a computer program. All fields were searched with both techniques. The efficiency was determined by placing artificial objects matched to the point-spread function of the images with motions similar to Uranus in the survey fields. Effective radii were calculated assuming the object had an albedo of 0.04 . The efficiency does not account for objects that would have been undetected because of the chip gaps.

The data were analyzed to find solar system bodies in two complementary ways. First, a computer algorithm was used to detect objects that appeared in all three images from one night and which had a motion consistent with being beyond the orbit of Jupiter (speeds less than $20^{\prime \prime} \mathrm{hr}^{-1}$ ). Second, all of the fields were examined by visually blinking them on a computer display screen for moving objects again with motions indicative of distances beyond Jupiter.

We determined the limiting magnitude of the survey in the absence of scattered light from Uranus by placing artificial objects in the fields matched to the point-spread function of the images and with motions mimicking that of Uranus. The brightnesses of the objects were binned by 0.1 mag and spanned the range from 25 th to 27 th magnitude. Results are shown in Figure 3 for both the visual blinking and the computer algorithm. The techniques gave similar results, though the visual blinking was slightly more efficient in detection. The $50 \%$ differential detection efficiency of the Uranian satellite survey occurred at an $R$-band limiting magnitude of about 26.1, which we take as the limiting magnitude of this survey. Scattered light from Uranus was only significant within 3.' 5 of the planet. Figure 4 shows the $50 \% R$-band limiting magnitude efficiency as a function of distance from Uranus in our survey. The scatteredlight results were determined in the same way as the nonscattered-light technique described above.

\section{RESULTS AND DISCUSSION}

Our survey detected, without prior knowledge of their positions, all of the then-known six irregular satellites of Uranus, as well as the two outermost regular satellites, Titania and Oberon. We also discovered the two new Uranian satellites S/2001 U2 and S/2003 U3 (Sheppard et al. 2003). S/2001 U2 has a 2001 designation because it had been detected but not confirmed as a Uranian satellite in 2001 (Holman et al. 2003). S/2001 U3 was confirmed as a Uranian irregular satellite after our survey (Marsden et al. 2003). This object went undetected in our Subaru data because it fell on a bright star during observations. In addition, six other objects near Uranus that turned 


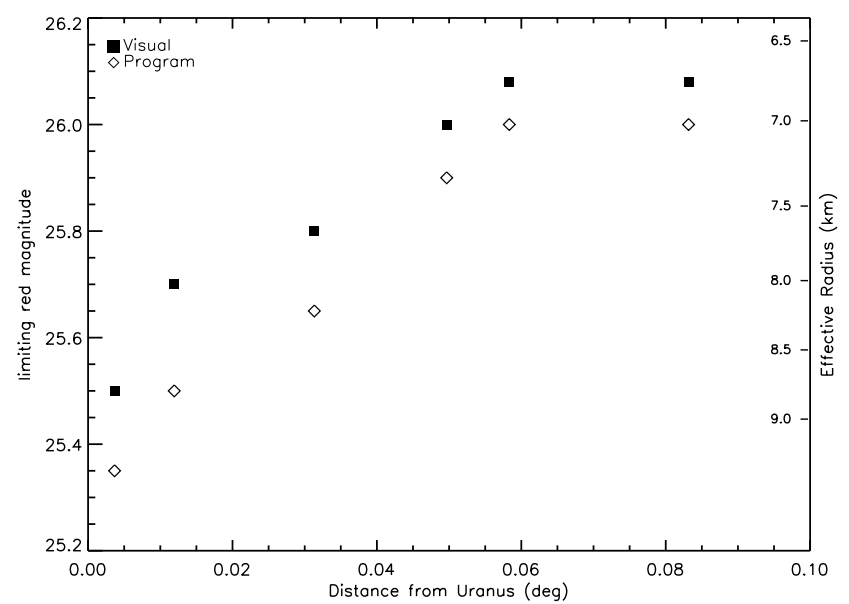

FIG. 4.- Red limiting magnitude (50\% detection efficiency) of the survey vs. distance from Uranus. Scattered light is only significant starting at about 3.'5 from Uranus. The calculation of the effective radius assumes an albedo of 0.04 .

out to be Centaurs, as well as hundreds of Kuiper belt objects, were discovered. A detailed report about the discovery of these other objects will be given in a future paper.

To determine the size limit of satellites detectable by the survey and the approximate sizes of the new satellites, we relate the apparent red magnitude, $m_{R}$, to the radius, $r$, through

$$
r=\left[\frac{2.25 \times 10^{16} R^{2} \Delta^{2}}{p_{R} \phi(\alpha)}\right]^{1 / 2} 10^{0.2\left(m_{\odot}-m_{R}\right)},
$$

in which $r$ is in kilometers, $R$ is the heliocentric distance in $\mathrm{AU}, \Delta$ is the geocentric distance in $\mathrm{AU}, m_{\odot}$ is the apparent red magnitude of the Sun $(-27.1), p_{R}$ is the geometric red albedo, $\phi(\alpha)$ is the phase function, and $\alpha$ is the phase angle $\left(\alpha=0^{\circ}\right.$ at opposition). For linear phase functions we use the notation $\phi(\alpha)=10^{-0.4 \beta \alpha}$, where $\beta$ is the "linear" phase coefficient. Using data from Table 2 and an albedo of 0.04 , we find that 26.1 mag corresponds to a satellite with radius of about $7 \mathrm{~km}$.

\subsection{Size and Population Distribution}

The cumulative luminosity function (CLF) describes the skyplane number density of objects brighter than a given magnitude. The CLF is conveniently described by

$$
\log \Sigma\left(m_{R}\right)=\alpha\left(m_{R}-m_{0}\right),
$$

where $\Sigma\left(m_{R}\right)$ is the number of objects brighter than $m_{R}, m_{0}$ is the magnitude zero point, and $\alpha$ describes the slope of the luminosity function. The CLF for Uranus's irregular satellites is shown in Figure 5. Our survey is complete to $m_{R}=26.1 \mathrm{mag}$ $(r>7 \mathrm{~km})$. For Uranus we find the best fit to the CLF for $m_{R}<26$ mag is $\alpha=0.20 \pm 0.04$ and $m_{0}=20.73 \pm 0.2$ (Fig. 5).

The points in a CLF are heavily correlated with one another, tending to give excess weight to the faint end of the distribution. The differential luminosity function (DLF) does not suffer from this problem. We plot the DLF using a bin size of 2 mag for all Uranian irregular satellites in Figure 6. We find that the DLF has a slope of $\alpha=0.16 \pm 0.05$ with $m_{0}=21.0 \pm 0.4$. Bin sizes of $1 \mathrm{mag}$ and $1.5 \mathrm{mag}$ give similar results. The uncertainty of the fit for the 2 mag bin size is estimated from the fits of these other bin sizes, which were larger than the uncertainty of the least-squares fit for the 2 mag bin size alone.

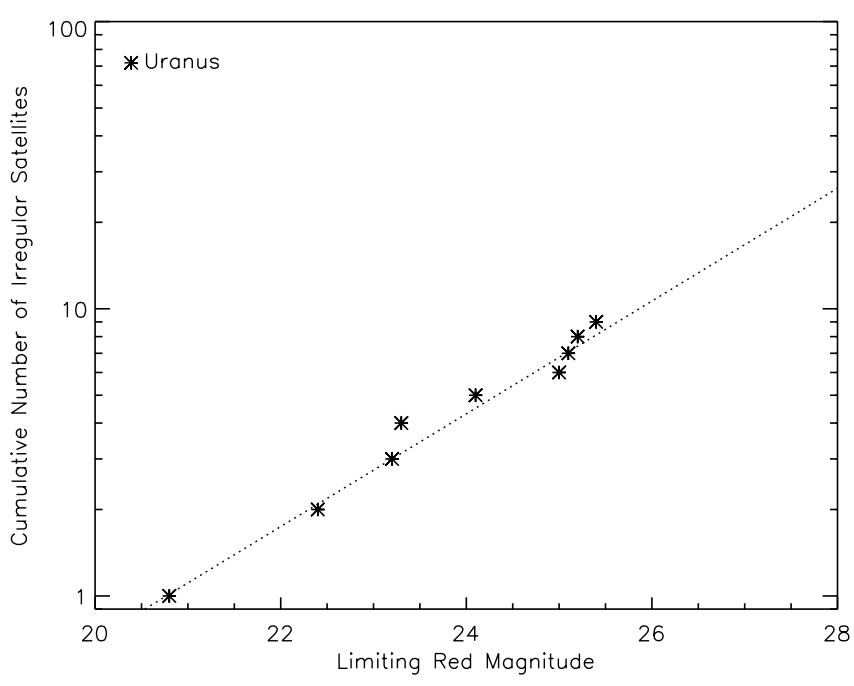

FIG. 5.-Cumulative luminosity function of the irregular satellites of Uranus. The dotted line is the slope of the CLF for Uranus's irregular satellites with $m_{R} \leq 26$ mag $(\alpha=0.20 \pm 0.04)$.

The luminosity functions represent the combined effects of the albedos, heliocentric distances, and size distributions of the objects. If we assume that all Uranian satellites are at the same heliocentric distance and that their albedos are similar, the DLF and CLF simply reflect the size distribution of the satellites. The objects appear to follow a single power-law size distribution for $r>7 \mathrm{~km}$. In order to model the irregular-satellite size distribution, we use a differential power-law radius distribution of the form $n(r) d r=\Gamma r^{-q} d r$, where $\Gamma$ and $q$ are constants, $r$ is the radius of the satellite, and $n(r) d r$ is the number of satellites with radii in the range $r$ to $r+d r$. The slope of the $\operatorname{DLF}(\alpha)$ and exponent of the size distribution $(q)$ are simply related as $q=5 \alpha+1$ if one assumes similar heliocentric distance and albedos for all satellites (Irwin et al. 1995). Using $\alpha=0.16 \pm 0.05$ for Uranus's irregular satellites, we find $q=$ $1.8 \pm 0.3$. This is similar to the value found by Kavelaars et al.

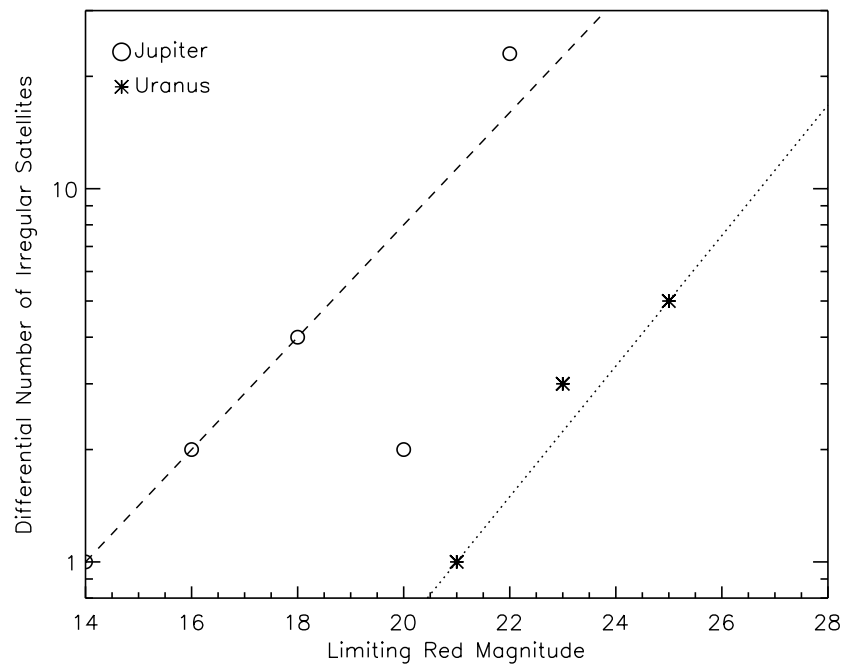

FIG. 6.-Differential luminosity function of the irregular satellites of Uranus and Jupiter. The slope for both planets is very similar, but because of Uranus's greater distance it is shifted about 6 mag to the right. The dotted line is the slope of the DLF for Uranus's irregular satellites with $r \geq 7 \mathrm{~km} \mathrm{(} \alpha=$ $0.16 \pm 0.05)$, and the dashed line is for Jupiter's irregular satellites in the same size range $(\alpha=0.18 \pm 0.05)$. A deficiency of satellites around Jupiter with magnitudes in the range $19<m_{R}<21.5(4 \mathrm{~km}<r<10 \mathrm{~km})$ can clearly be seen, as described in Sheppard \& Jewitt (2003). 
(2004) and identical to the $q=1.9 \pm 0.3$ found for the irregular satellites of Jupiter in the same size range (Sheppard \& Jewitt 2003).

In comparison, collisional equilibrium gives $q \sim 3.5$ (Dohnanyi 1969) and nonfamily small asteroids have $q \sim 2.0-$ 2.5 (Cellino et al. 1991), while large Kuiper belt objects (KBOs) have $q=4.2 \pm 0.5, \alpha=0.64 \pm 0.1$, and $m_{0}=23.23 \pm 0.15$ (Trujillo et al. 2001) and Centaurs have KBO-like slope with $m_{0}=24.6 \pm 0.3$ (Sheppard et al. 2000). The small Jovian Trojans $(r<30 \mathrm{~km})$ have $q=3.0 \pm 0.3$, while the larger Trojans show a steeper slope of $q=5.5 \pm 0.9$ (Jewitt et al. 2000). Large members of asteroid families have been found to usually have $q \geq 4$ (Tanga et al. 1999), while the smaller members $(r<5 \mathrm{~km})$ may have shallower distributions (Morbidelli et al. 2003). If $q>3$ most of the collisional cross section lies in the smallest objects, while for $q>4$ most of the mass lies in small bodies. The mass and cross section of Uranus's irregular satellites are both dominated by the few largest objects.

\subsection{Comparison of the Uranian and Jovian Systems}

The Jovian and Uranian satellite DLFs are compared in Figure 6. Fitting the Jovian DLF (which is complete to around $r \sim 3 \mathrm{~km}$; Sheppard \& Jewitt 2003) over the same size range as that used previously for Uranus $(r>7 \mathrm{~km})$, we find a slope of $\alpha=0.18 \pm 0.05$ with a zero-point magnitude $m_{0}=14.0 \pm$ 0.4 . The measured slope is compatible with $\alpha=0.16 \pm 0.05$, as found for the Uranian satellites, within the statistical uncertainties. We conclude that the size distribution indices of the irregular satellites at Uranus and Jupiter are remarkably similar for $r>7 \mathrm{~km}$, and both are quite different from the (steeper) distribution that would be measured, for example, among the main-belt asteroids. Jupiter's irregular satellites appear depleted in the $4-10 \mathrm{~km}$ size range relative to an extrapolation of a power law fitted at larger sizes (Sheppard \& Jewitt 2003). We would need deeper survey observations, to $m_{R} \sim 27.2 \mathrm{mag}$, to determine whether the Uranian population shows a similar depletion.

The difference between the Jovian and Uranian DLF magnitude zero points is $\Delta m_{0}=7.0 \pm 0.6 \mathrm{mag}$. This is to be compared with the magnitude difference expected from the inverse square law and the different distances to the two planets. The expected magnitude difference is $\Delta m=5 \log \left[R_{\mathrm{U}}\left(R_{\mathrm{U}}-\right.\right.$ $\left.1) / R_{\mathrm{J}}\left(R_{\mathrm{J}}-1\right)\right]$, where $R_{\mathrm{J}}=5.3 \mathrm{AU}$ and $R_{\mathrm{U}}=20.0 \mathrm{AU}$ are the heliocentric distances to the two planets and opposition geometry is assumed. Substituting, we obtain $\Delta m=6.1 \mathrm{mag}$, which is different from $\Delta m_{0}$ by only about $1.5 \sigma$. In this sense, the smaller number of known irregular satellites at Uranus seems to be an artifact of the greater distance.

To emphasize these points, we show the cumulative size distributions of the Jovian and Uranian irregular satellites in Figure 7. Uranus has nine satellites with $r \geq 7 \mathrm{~km}$ (about the completeness level), while Jupiter has eight. In terms of the size distributions and total populations, the irregular-satellite systems of Uranus and Jupiter are very similar. If we assume the size distribution of Uranus's irregular satellites extends down to radii of about $1 \mathrm{~km}$, we would expect about $75 \pm 30$ irregular satellites of this size or larger.

Several competing processes could influence the size distribution of the satellites. The larger objects may retain some memory of the production function, as is apparently the case with the nearby Jovian Trojans, for which the size distribution is steeper below $r \sim 30 \mathrm{~km}$ than above it (Jewitt et al. 2000). Small satellites could be lost to gas drag, leading to a flattening of the size distribution. This is more likely at gas giant Jupiter

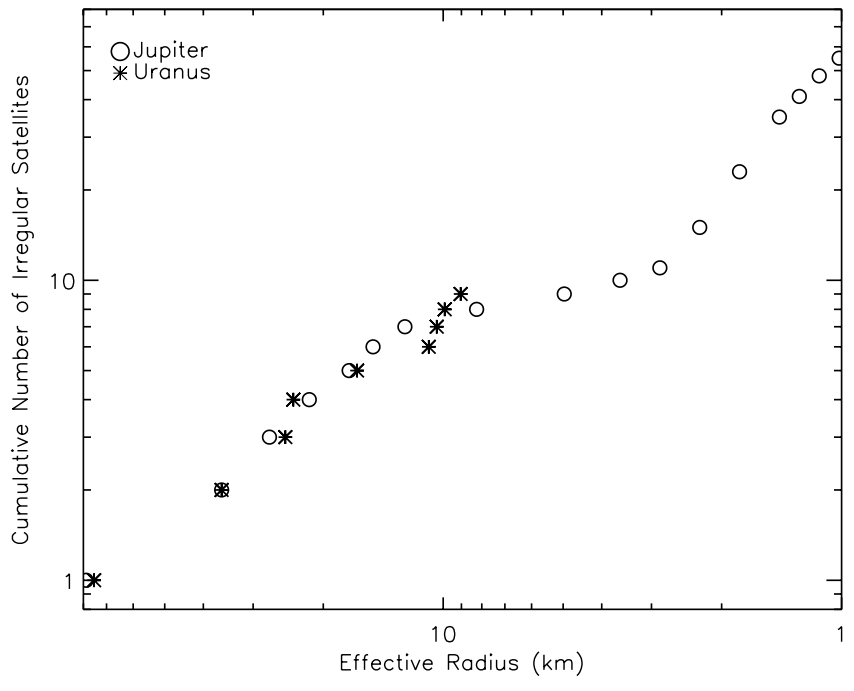

FIG. 7.-Cumulative radius function for the irregular satellites of Uranus and Jupiter. This figure directly compares the sizes of the satellites of the two planets assuming both satellite populations have albedos of about 0.04 . The two planets have statistically similar size distributions of irregular satellites $(q \sim 2)$ for a size range of $r \geq 7 \mathrm{~km}$.

than at ice giant Uranus, where much less gas is thought to have been available during the accretion epoch. Collisions between satellites and with interplanetary projectiles would lead to the production of many small fragments. Given that fragment velocity and size are inversely related, it is natural to expect that the smaller objects produced collisionally would be lost (the escape velocity from Uranus at the semimajor axis of $\mathrm{S} / 2001 \mathrm{U} 2$ is only $0.8 \mathrm{~km} \mathrm{~s}^{-1}$ ), again leading to a flattened size distribution. Collisional scenarios, in general, require higher collision rates than now prevail in the solar system. Perhaps the irregular satellites were originally much more numerous than now. Separately, we know that the flux of planet-crossing projectiles was much higher between the epochs of planet formation and the end of the terminal bombardment phase at about 3.9 Gyr.

\subsection{Orbital Element Distribution}

Table 3 lists some of the properties of the known irregular satellites of Uranus. Figures 8 and 9 compare the semimajor axes with inclination and eccentricity, respectively, for all known irregular satellites of the planets. The figures show that the ice giants Uranus and Neptune have the smallest known irregular-satellite systems, in units of Hill radii. In the case of Uranus, one contributing factor may be that its regular-satellite system is much less massive and does not extend as far from the planet as those of the gas giant planets Jupiter and Saturn. Thus, interactions between Uranus's regular and irregular satellites are less important as a clearing mechanism. We also note that Uranus has the smallest $a_{\text {crit }}$ of any of the giant planets (Table 1). Neptune's satellite system may have been severely disrupted by the unusually large retrograde satellite Triton (Goldreich et al. 1989).

Figure 8 suggests that the Uranian irregular satellites may be grouped in semimajor-axis versus eccentricity phase space. The four retrograde irregular satellites closest to Uranus $(\mathrm{S} / 2001$ U3, Caliban, Stephano, and Trinculo; semimajor axes of $a<$ $0.15 r_{\mathrm{H}}$ ) have eccentricities $e \sim 0.2$, while the four retrograde irregular satellites (Sycorax, Prospero, Setebos, and S/2001 U2) with $a>0.15 r_{\mathrm{H}}$ have $e \sim 0.5$. 
TABLE 3

Physical and Orbital Properties of Uranus's Irregular Satellites

\begin{tabular}{|c|c|c|c|c|c|c|c|c|c|c|}
\hline Name & $\begin{array}{c}a^{\mathrm{a}} \\
\left(10^{3} \mathrm{~km}\right)\end{array}$ & $\begin{array}{c}i^{\mathrm{b}} \\
(\mathrm{deg})\end{array}$ & $e^{\mathrm{c}}$ & $\begin{array}{l}\text { Peri. }^{\mathrm{d}} \\
(\mathrm{deg})\end{array}$ & $\begin{array}{l}\mathrm{Node}^{\mathrm{e}} \\
(\mathrm{deg})\end{array}$ & $\begin{array}{c}M^{\mathrm{f}} \\
(\mathrm{deg})\end{array}$ & $\begin{array}{c}\text { Period }^{g} \\
\text { (days) }^{-}\end{array}$ & $m_{R}^{\mathrm{h}}$ & $\begin{array}{c}r^{\mathrm{i}} \\
(\mathrm{km})\end{array}$ & Year $^{\mathrm{j}}$ \\
\hline \multicolumn{11}{|c|}{ Low $a$, low $e$ : } \\
\hline 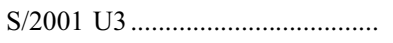 & 4276 & 145 & 0.15 & 125 & 93 & 91 & 266.6 & 25.0 & 11 & 2002 \\
\hline XVI Caliban (S/1997 U1) ........... & 7231 & 141 & 0.16 & 343 & 164 & 163 & 579.7 & 22.4 & 36 & 1997 \\
\hline XX Stephano (S/1999 U2) .......... & 8004 & 144 & 0.23 & 19 & 188 & 82 & 677.4 & 24.1 & 16 & 1999 \\
\hline XXI Trinculo (S/2001 U1) .......... & 8504 & 167 & 0.22 & 160 & 195 & 22 & 759.0 & 25.4 & 9 & 2001 \\
\hline \multicolumn{11}{|c|}{ High $a$, high $e$ : } \\
\hline XVII Sycorax (S/1997 U2) ......... & 12179 & 159 & 0.52 & 20 & 260 & 170 & 1288.3 & 20.8 & 75 & 1997 \\
\hline XVIII Prospero (S/1999 U3) ....... & 16256 & 152 & 0.45 & 176 & 317 & 181 & 1977.3 & 23.2 & 25 & 1999 \\
\hline XIX Setebos (S/1999 U1) ............. & 17418 & 158 & 0.59 & 1.5 & 248 & 126 & 2234.8 & 23.3 & 24 & 1999 \\
\hline 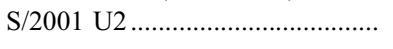 & 20901 & 170 & 0.37 & 160 & 216 & 235 & 2823.4 & 25.1 & 10 & 2003 \\
\hline \multicolumn{11}{|c|}{ Prograde: } \\
\hline 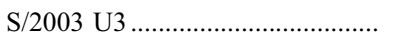 & 14345 & 57 & 0.66 & 89 & 3.5 & 322 & 1694.8 & 25.2 & 10 & 2003 \\
\hline
\end{tabular}

Note.—Orbital data are from R. Jacobson at JPL (http://ssd.jpl.nasa.gov/satelem.html). Fits are over a $1000 \mathrm{yr}$ time span.

${ }^{a}$ Mean semimajor axis with respect to Uranus.

${ }^{b}$ Mean inclination of orbit with respect to the ecliptic.

c Mean eccentricity.

d Argument of periapsis.

e Longitude of the ascending node.

${ }^{\mathrm{f}}$ Mean anomaly.

g Orbital period of satellite around Uranus.

h Apparent red (0.65 $\mu \mathrm{m}$ wavelength) magnitude.

${ }^{i}$ Radius of satellite assuming a geometric albedo of 0.04 .

${ }^{\mathrm{j}}$ Year in which observations determined that the object was a satellite.

To judge the significance of these two retrograde groups, we performed several statistical tests. The retrograde loweccentricity, low semimajor axis group (the Caliban group) has a mean eccentricity of $0.19 \pm 0.02$ and mean semimajor axis of $(7.0 \pm 0.9) \times 10^{6} \mathrm{~km}$, while the retrograde high semimajor axis and eccentricity group (the Sycorax group) has $0.52 \pm 0.05$ and
$(16.7 \pm 1.8) \times 10^{6} \mathrm{~km}$, respectively. Student's $t$-test (with $7 \mathrm{de}-$ grees of freedom) gave a $t$-statistic of 6.0 with a significance of $99.8 \%$ for the difference in their mean eccentricities and a $t$-statistic of 4.8 with a significance of $99.4 \%$ for the difference in their mean semimajor axes. The $t$-test suggests that the two groups are significant but makes the unjustified assumption that

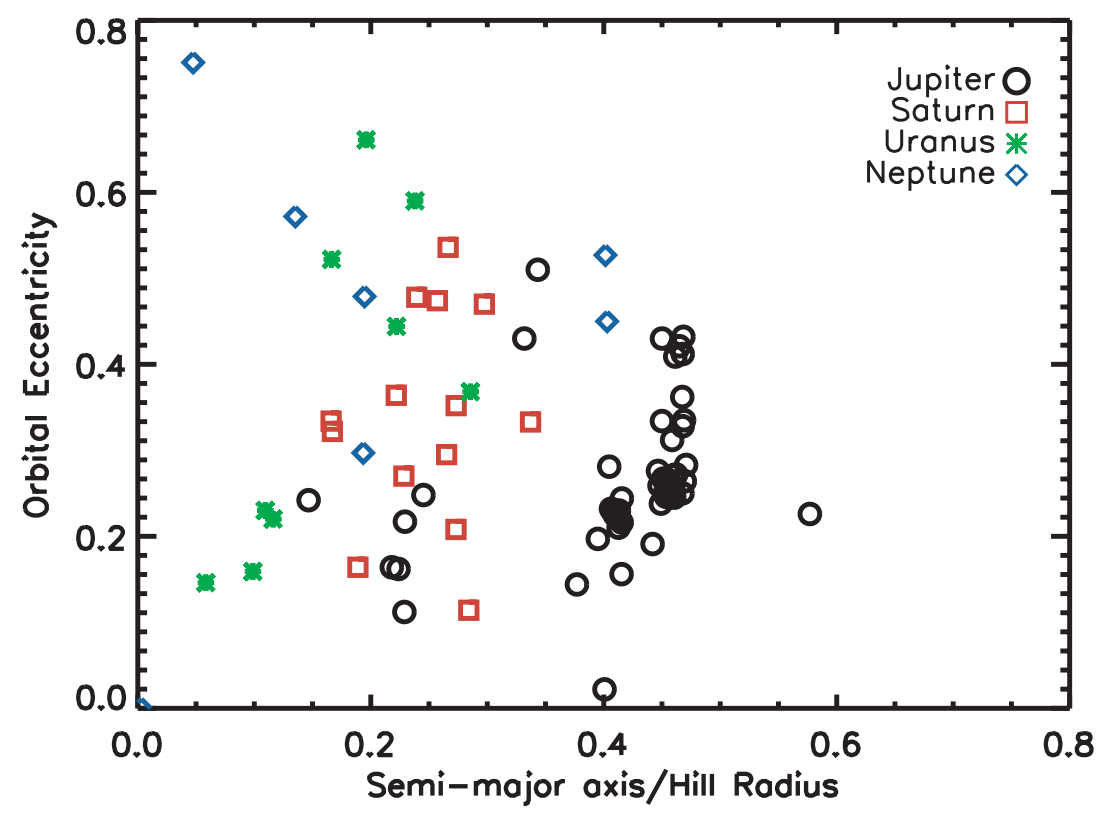

FIG. 8.-Mean eccentricity comparison between the known irregular satellites of the giant planets. The horizontal axis is the ratio of the satellite's mean semimajor axis to its respective planet's Hill radius. The vertical axis is the mean eccentricity of the satellite. All giant planets independent of their mass or formation appear to have similar irregular-satellite systems. Except for Neptune's "unusual" Triton and Nereid, Uranus appears to have the closest irregular satellites in terms of Hill radii of the giant planets. 


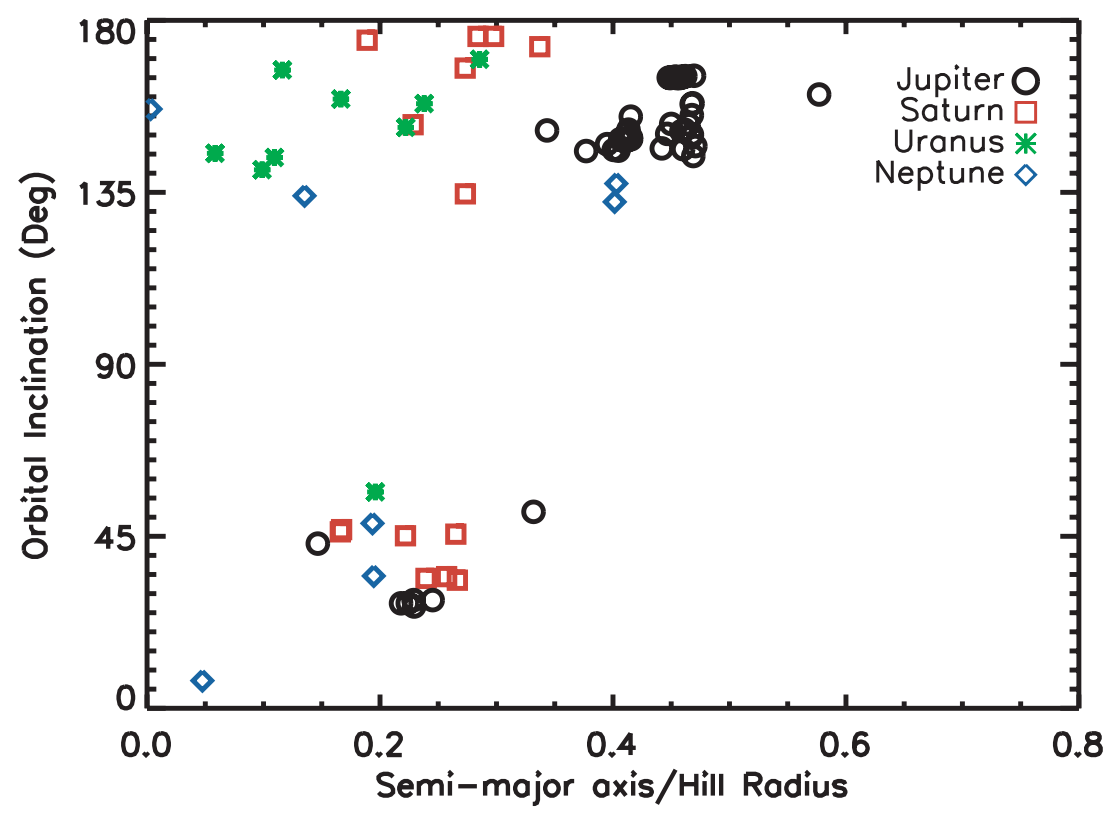

FIG. 9.- Same as Fig. 8, except here mean inclination to the ecliptic is used instead of eccentricity for the vertical axis.

the eccentricity and semimajor axes are normally distributed. Therefore, we used the more stringent nonparametric MannWhitney $U$-test and a permutation test (see Siegel \& Castellan 1988) to assess the significance of the two groups. Both found that the groupings of the eccentricity were statistically significant at or above the $\geq 3 \sigma(99.7 \%)$ level of confidence.

Figure 10 shows the two retrograde groups in semimajor-axis versus eccentricity space. It can clearly be seen that the more distant satellites have larger eccentricities, as was also noted by Kavelaars et al. (2004). The significance of a linear fit to the data is only at the $\sim 97 \%(<3 \sigma)$ level and thus less significant than the two groupings. Possible reasons for higher eccentricities more distant from the planet are that the closer satellites would be unstable to perturbations by the much larger regular satellites of Uranus if they had large eccentricities, and that the more distant satellites are susceptible to solar and planetary perturbations. In Figure 10 we plot several lines of constant periapse from Uranus.

The orbital velocities of the irregular satellites around Uranus range from 300 to $1100 \mathrm{~m} \mathrm{~s}^{-1}$ (Kessler 1981). The relative velocities among the satellites are typically much smaller. Velocity differences among Caliban, Stephano, and Trinculo are comparable to Caliban's escape velocity, $\sim 40 \mathrm{~m} \mathrm{~s}^{-1}$. The retrograde high-eccentricity objects have relative velocities comparable to Sycorax's escape velocity of $\sim 80 \mathrm{~m} \mathrm{~s}^{-1}$. For comparison, Jupiter's irregular satellites were found to be grouped in semimajor-axis and inclination phase space with their relative velocities within a group about $30 \mathrm{~m} \mathrm{~s}^{-1}$, while group velocities relative to each other were over $200 \mathrm{~m} \mathrm{~s}^{-1}$ (Sheppard \& Jewitt 2003). As at Jupiter, Uranus's possible two retrograde groups have relative velocities of over $100 \mathrm{~m} \mathrm{~s}^{-1}$, while members within a group have velocities comparable to the largest members' escape velocity. As at Jupiter, the dynamical groupings suggest formation from parent objects that were collisionally shattered. A simple particle-in-a-box calculation shows that collisions between the currently known or predicted outer satellite population of Uranus would occur on timescales $\left(\sim 10^{10} \mathrm{yr}\right)$ longer than the age of the solar system. Fragmentation could have occurred from collisions with objects in heliocentric orbits (principally comets around the heavy bom- bardment period; Sheppard \& Jewitt 2003) or other now-defunct satellites of the planet (Nesvorný et al. 2004). Jupiter's irregularsatellite orbital velocities $\left(>2200 \mathrm{~m} \mathrm{~s}^{-1}\right)$ are much greater than Uranus's, and thus any collisional processing would have been much more violent compared with collisions around Uranus.

Except for the distinct prograde irregular S/2003 U3, with an orbital inclination of $57^{\circ}$ to the ecliptic compared with the eight irregular satellites between $140^{\circ}$ and $170^{\circ}$, there are no obvious tight groupings in semimajor-axis versus inclination phase space as was found around Jupiter (Fig. 9). We do note that the majority of the low-eccentricity retrograde satellites have inclinations near $142^{\circ}$, while the retrograde high-eccentricity satellites are closer to $156^{\circ}$ in inclination (Table 3). The intermediate

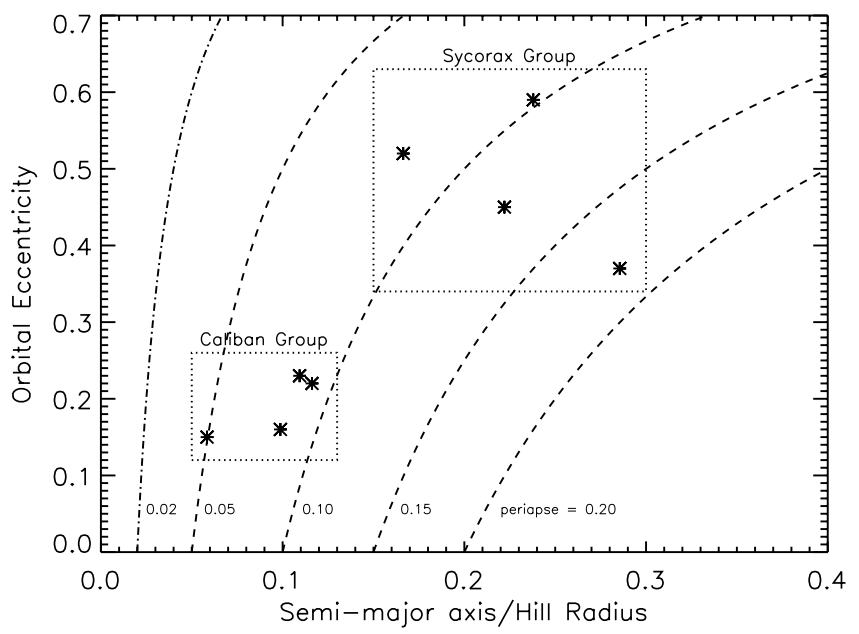

FIG. 10.-Orbital eccentricity vs. mean semimajor axis in units of the Hill radius for retrograde Uranus irregular satellites only. There is a trend for satellites to have a larger eccentricity if they are more distant from Uranus. Boxes show and are named after the largest member of the two possible groupings discussed in the text. A simple linear fit gives a slope of $2.0 \pm 0.7$ and a $y$-intercept at $0.0 \pm 0.1$, but the Pearson correlation coefficient is only 0.76 , corresponding to a statistical significance of less than $3 \sigma$. The two groupings are statistically more significant than the linear fit. Dashed lines show constant periapse distances of $0.02,0.05,0.10,0.15$, and 0.20 Hill radii, respectively. The regular satellites of Uranus are found inside 0.02 Hill radii. 
inclinations $60^{\circ}<i<140^{\circ}$ are devoid of known satellites, consistent with instabilities caused by the Kozai instability (Kozai 1962; Carruba et al. 2002; Nesvorný et al. 2003). In this instability, solar perturbations at apoapse cause the satellites at high inclinations to acquire large eccentricities that eventually lead to collisions with the planet or a regular satellite or loss from the Hill sphere in $10^{7}-10^{9}$ years (Carruba et al. 2002; Nesvorný et al. 2003).

We find no clear size versus semimajor axis, inclination or eccentricity correlations for Uranus's irregular satellites, as may be expected if significant gas drag was present in the past. Of the two largest irregular satellites around Uranus, one of each is in the two possible eccentricity groups. Caliban is relatively close to the planet with a low eccentricity, while Sycorax is with the distant, higher eccentricity irregular satellites (Table 3).

At Uranus there are many more known retrograde (8) than prograde (1) outer satellites. Jupiter also has an overabundance of known retrograde outer satellites (48 retrograde vs. 7 prograde). These asymmetries are greatly diminished if we compare numbers of satellite groups (about three to four retrograde versus three prograde groups at Jupiter and possibly one or two retrograde and one prograde group at Uranus). Given this, and the fact that the statistics of the groups remain poor (especially at Uranus), we cannot currently use the relative numbers of retrograde and prograde objects to constrain the mode of capture.

\section{SUMMARY}

1. We have conducted a deep imaging survey of $3.5 \mathrm{deg}^{2}$ around Uranus covering most of the region in which long-term stable orbits are possible. The effective limiting red magnitude of the survey is $m_{R}=26.1$ ( $50 \%$ detection efficiency). This corresponds to objects of about $7 \mathrm{~km}$ in radius if assuming a 0.04 geometric albedo.

2. We detected, without prior knowledge of their positions, all previously known irregular satellites in addition to two new irregular satellites (S/2001 U2 and S/2003 U3). The latter is Uranus's first and, so far, only known prograde irregular satellite.

3. The differential size distribution of the irregular satellites approximates a power law with an exponent $q=1.8 \pm 0.3$ (radii greater than $7 \mathrm{~km}$ ). In this relatively flat distribution, the cross section and mass are dominated by the few largest members. The size distribution is essentially the same as found independently for the irregular satellites of the gas giant Jupiter.

4. The Jovian and Uranian irregular-satellite populations, when compared with a given limiting size, are similar. For example, the number of satellites larger than $7 \mathrm{~km}$ in radius (albedo 0.04 assumed) is nine at Uranus, compared with eight at Jupiter. The similarity is remarkable given the different formation scenarios envisioned for these two planets.

5. The orbital parameters of the satellites are unrelated to their sizes.

6. We tentatively define two groups of retrograde irregular satellites in semimajor-axis versus eccentricity phase space. The four satellites of the inner retrograde group (Caliban, S/2001 U3, Stephano, and Trinculo) have semimajor axes of less than 0.15 Hill radii and moderate eccentricities $(\sim 0.2)$, while the four members of the outer retrograde group (Sycorax, Prospero, Setebos, and S/2001 U2) have larger semimajor axes $(>0.15$ Hill radii) with higher eccentricities $(\sim 0.5)$. Unlike at Jupiter, the currently known retrograde irregular satellites are not tightly grouped in semimajor-axis versus inclination space.

We thank Richard Wainscoat for confirming coordinates for Gemini, and the Gemini staff for running the queue servicing mode with GMOS. We are grateful to Brian Marsden and Bob Jacobson for orbital calculations relating to the satellites and to the anonymous referee for a careful review. This work was supported by a grant from NASA to D. J.
Beaugé, C., Roig, F., \& Nesvorný, D. 2002, Icarus, 158, 483

Boss, A. P. 2001, ApJ, 563, 367 2003, ApJ, 599, 577

Brunini, A., Parisi, M. G., \& Tancredi, G. 2002, Icarus, 159, 166

Burns, J. A. 1986, in Satellites, ed. J. A. Burns \& M. S. Matthews (Tucson: Univ. Arizona Press), 117

Carruba, V., Burns, J. A., Nicholson, P. D., \& Gladman, B. J. 2002, Icarus, 158, 434 (erratum 162, 230 [2003])

Cellino, A., Zappalà, V., \& Farinella, P. 1991, MNRAS, 253, 561

Colombo, G., \& Franklin, F. A. 1971, Icarus, 15, 186

Dohnanyi, J. 1969, J. Geophys. Res., 74, 2531

Gladman, B., Kavelaars, J. J., Holman, M., Petit, J.-M., Scholl, H., Nicholson, P., \& Burns, J. A. 2000, Icarus, 147, 320 (erratum 148, 320)

Goldreich, P., Murray, N., Longaretti, P. Y., \& Banfield, D. 1989, Science, 245,500

Greenberg, R. 1974, Icarus, 23, 51

Hamilton, D. P., \& Krivov, A. V. 1997, Icarus, 128, 241

Q2 Hénon, M. 1970, A\&A, 9, 24

Heppenheimer, T. A., \& Porco, C. 1977, Icarus, 30, 385

Holman, M., Gladman, B., Marsden, B. G., Sheppard, S. S., \& Jewitt, D. C. 2003, IAU Circ. 8213

Hook, I., et al. 2003, Proc. SPIE, 4841, 1645

Irwin, M., Tremaine, S., \& Żytkow, A. N. 1995, AJ, 110, 3082

Jewitt, D., Sheppard, S., \& Porco, C. 2004, in Jupiter: The Planet, Satellites, and Magnetosphere, ed. F. Bagenal, T. E. Dowling, \& W. B. McKinnon (Cambridge: Cambridge Univ. Press), in press

Jewitt, D. C., Trujillo, C. A., \& Luu, J. X. 2000, AJ, 120, 1140

Kavelaars, J. J., et al. 2004, Icarus, 169, 474

Kessler, D. J. 1981, Icarus, 48, 39

Korycansky, D. G., Bodenheimer, P., Cassen, P., \& Pollack, J. B. 1990, Icarus, 84,528

Kozai, Y. 1962, AJ, 67, 591

\section{REFERENCES}

Kuiper, G. P. 1956, Vistas Astron., 2, 1631

1961, in Planets and Satellites, ed. G. P. Kuiper \& B. M. Middlehurst (Chicago: Univ. Chicago Press), 575

Landolt, A. U. 1992, AJ, 104, 340

Lissauer, J. J., Pollack, J. B., Wetherill, G. W., \& Stevenson, D. J. 1995, in Neptune and Triton, ed. D. P. Cruikshank (Tucson: Univ. Arizona Press), 37 Marsden, B. G., Holman, M., Gladman, B., Rousselot, P., \& Mousis, O. 2003, IAU Circ. 8216

Miyazaki, S., et al. 2002, PASJ, 54, 833

Morbidelli, A., Nesvorný, D., Bottke, W. F., Michel, P., Vokrouhlický, D., \& Tanga, P. 2003, Icarus, 162, 328

Nesvorný, D., Alvarellos, J. L. A., Dones, L., \& Levison, H. F. 2003, AJ, 126,398

Nesvorný, D., Beaugé, C., \& Dones, L. 2004, AJ, 127, 1768

Peale, S. J. 1999, ARA\&A, 37, 533

Pollack, J. B., Burns, J. A., \& Tauber, M. E. 1979, Icarus, 37, 587

Pollack, J. B., Hubickyj, O., Bodenheimer, P., Lissauer, J. J., Podolak, M., \& Greenzweig, Y. 1996, Icarus, 124, 62

Sheppard, S. S., \& Jewitt, D. C. 2003, Nature, 423, 261

Sheppard, S. S., Jewitt, D. C., Marsden, B. G., Holman, M., \& Kavelaars, J. 2003, IAU Circ. 8217

Sheppard, S. S., Jewitt, D. C., Trujillo, C. A., Brown, M. J. I., \& Ashley, M. C. B. 2000, AJ 120, 2687

Siegel, S., \& Castellan, N. J., Jr. 1988, Nonparametric Statistics for the Behavioral Sciences (2nd ed.; New York: McGraw-Hill)

Slattery, W. L., Benz, W., \& Cameron, A. G. W. 1992, Icarus, 99, 167

Tanga, P., Cellino, A., Michel, P., Zappalà, V., Paolicchi, P., \& Dell'Oro, A. 1999, Icarus, 141, 65

Thommes, E. W., Duncan, M. J., \& Levison, H. F. 2002, AJ, 123, 2862

Trujillo, C. A., Jewitt, D. C., \& Luu, J. X. 2001, AJ, 122, 457

Tsui, K. 2000, Icarus, 148, 139 


\section{AUTHOR QUERIES}

Q1 Au: Added Goldreich et al. (1989), Sheppard et al. (2000) entries correct?

Q2 Au: Hénon (1970), Jewitt et al. (2004), Kuiper (1961) are not cited in the text; please either cite or delete. 